\title{
Use of the Inertial Dissipation Method for Calculating Turbulent Fluxes from Low-Level Airborne Measurements
}

\author{
Pierre Durand, Leonardo de Sa, Aimé Druilhet and Frédérique Said \\ Laboratoire d'Aérologie, Université Paul Sabatier, Toulouse, France
}

(Manuscript received 23 February 1990, in final form 3 October 1990)

\section{ABSTRACT}

\begin{abstract}
Airborne measurements are currently used for computing turbulence fluxes of heat and momentum. The method generally used is the eddy correlation technique, which requires sophisticated equipments to calculate the absolute velocities of the air. We used the well-known inertial dissipation method to calculate the turbulent fluxes of heat and momentum from low-level airborne measurements. This only requires knowledge of inertial subrange characteristics of velocity and scalars. The method was validated by comparing dissipation fluxes with those computed by the eddy correlation method. The agreement between the two is very good, particularly for heat fluxes. Last, it is shown how the turbulent kinetic energy dissipation rate can be easily calculated, using a single measurement (the attack angle by example), and therefore how turbulent fluxes can be simply calculated from low level airborne measurements.
\end{abstract}

\section{Introduction}

Turbulent fluxes of momentum and heat in the atmospheric surface layer can be measured using four methods: (i) the eddy-correlation method, (ii) the inertial dissipation method, (iii) the profile method and (iv) the bulk aerodynamic method. Method (i) is generally considered the method of reference, since it allows direct calculation of flux from measurement of turbulent fluctuations. The profile method generally gives good results for the continental surface layer, within ranges of stability where the universal profile functions are well known. The bulk aerodynamic method is frequently employed over the sea, as it only requires measurement of mean characteristics: wind at the reference level, and variations in temperature and water content between the surface and the reference level. It does, however, require knowledge of the aerodynamic coefficients for the momentum and sensible and latent heat $\left(C_{d}, C_{t}\right.$ and $\left.C_{q}\right)$ over which there is not yet complete agreement, particularly for very strong or very slight winds.

With airborne measurements, it is generally the eddy-correlation method that is used to calculate turbulent flux. It is true that this method requires no prior hypotheses for application and is applicable at any level.

"On leave from "Instituto de Pesquisas Espaciais" (INPE), Sao José dos Campos, Brazil.

Corresponding author address: Dr. Pierre Durand, Laboratoire d'Aérologie, Université Paul Sabatier, 118, route de Narbonne, 31062 Toulouse Cedex, France.
Its main drawback is that it requires knowledge of the absolute vertical velocity of the air, usually calculated from combined aerodynamic measurements and an inertial platform. This calculation is usually complicated by low-frequency drift, requiring high-pass filtering for computation of covariances.

The aim of this paper is to show how, using lowlevel airborne measurements, it is possible to employ a method simpler than the eddy-correlation method to calculate turbulent flux-namely, the inertial dissipation method.

\section{Principle of the inertial dissipation method}

The principle behind the method consists in calculating turbulent flux via measurement of inertial subrange characteristics of the spectra for velocity, temperature and water content. These characteristics are related to flux through the equations for the evolution of turbulent kinetic energy (TKE) and temperature and water content variances:

$$
\begin{aligned}
\frac{\partial \bar{e}}{\partial t}+\overline{u^{\prime} w^{\prime}} \frac{\partial \bar{u}}{\partial z}+\overline{v^{\prime} w^{\prime}} \frac{\partial \bar{v}}{\partial z}+\frac{\partial}{\partial z} \overline{w^{\prime} e^{\prime}} \\
+\frac{1}{\rho} \frac{\partial}{\partial z}\left(\overline{p^{\prime} w^{\prime}}\right)-\frac{g}{T} \overline{w^{\prime} \theta_{v}^{\prime}}+\epsilon=S_{e} \\
\frac{1}{\frac{\partial \theta^{\prime 2}}{\partial t}}+\overline{w^{\prime} \theta^{\prime}} \frac{\partial \bar{\theta}}{\partial z}+\frac{1}{2} \frac{\partial}{\partial z} \overline{w^{\prime} \theta^{\prime 2}}+N_{\theta}=S_{\theta} \\
\frac{1}{\frac{1}{2}} \frac{\partial q^{\prime 2}}{\partial t}+\overline{w^{\prime} q^{\prime}} \frac{\partial \bar{q}}{\partial z}+\frac{1}{2} \frac{\partial}{\partial z} \overline{w^{\prime} q^{\prime 2}}+N_{q}=S_{q}
\end{aligned}
$$

where $e$ denotes TKE; $u, v$ and $w$ the longitudinal, transversal and vertical wind components; $p$ the pres- 
sure; $\rho$ the density; $\theta_{v}$ the virtual potential temperature; $g / T$ the buoyancy parameter; $\epsilon$ the dissipation rate of TKE; $N_{\theta}$ and $N_{q}$ the half variance destruction rate in temperature and water content; $S_{\rho}, S_{\theta}$ and $S_{q}$ local sources and sinks for $e, \overline{\theta^{\prime 2}}$ and $\overline{q^{\prime 2}}$ (radiative effects, evaporation-condensation, etc. . . .).

The principle of the method consists in employing a simplified form of these equations, in particular neglecting the terms for local sources and sinks, vertical turbulent transport of variance and the term related to pressure fluctuation ( for a discussion of the validity of these hypotheses see, for example, Large and Pond 1982; Dyer and Hicks 1982; Fairall and Larsen 1986). The method also employs the universal formulations of nondimensional gradients for the surface layer. We worked under moderately unstable conditions (see section 3), and used the formulations of Dyer and Hicks (1970)

$$
\begin{aligned}
& \phi_{m}\left(\frac{z}{L}\right)=\left(1-16 \frac{z}{L}\right)^{-1 / 4}=\left(\frac{\partial \bar{u}}{\partial z}\right) \frac{k z}{u_{*}} \\
& \phi_{\theta}\left(\frac{z}{L}\right)=\left(1-16 \frac{z}{L}\right)^{-1 / 2}=-\left(\frac{\partial \bar{\theta}}{\partial z}\right) \frac{k z u_{*}}{\overline{w^{\prime} \theta_{0}^{\prime}}} \\
& \phi_{q}\left(\frac{z}{L}\right)=\left(1-16 \frac{z}{L}\right)^{-1 / 2}=-\left(\frac{\partial \bar{q}}{\partial z}\right) \frac{k z u_{*}}{\overline{w^{\prime} q_{0}^{\prime}}}
\end{aligned}
$$

where $L$ is the Monin-Obukhov length $\left\{L=-u_{*}^{3}\right.$ \} $\left.\left[k \beta\left(\overrightarrow{w^{\prime} \theta_{0}^{\prime}}\right)\right]\right\}, k$ is the von Kármán constant $(=0.4)$. Equations (1) to (3) thus take the following simplified form:

$$
\begin{gathered}
\phi_{m}\left(\frac{z}{L}\right)-\frac{z}{L}=\frac{\epsilon k z}{u_{*}^{3}} \\
\phi_{\theta}\left(\frac{z}{L}\right)=\frac{N_{\theta} k z u_{*}}{\overline{w^{\prime} \theta^{\prime 2}}} \\
\phi_{q}\left(\frac{z}{L}\right)=\frac{N_{q} k z u_{*}}{\overline{w^{\prime} q^{\prime 2}},}
\end{gathered}
$$

thereby allowing calculation of heat and momentum turbulent flux from the measurement of $\epsilon, N_{\theta}$ and $N_{q}$. These equations were solved using an iterative process, starting from neutral stability. $\epsilon, N_{\theta}$ and $N_{q}$ were calculated in the inertial subrange by applying Kolmogorov's formulation of the velocity, temperature and water content spectra:

$$
\begin{aligned}
S_{u_{i}}(K) & =\alpha_{i} \epsilon^{2 / 3} K^{-5 / 3} \\
S_{\theta}(K) & =\beta_{\theta} \epsilon^{-1 / 3} N_{\theta} K^{-5 / 3} \\
S_{q}(K) & =\beta_{q} \epsilon^{-1 / 3} N_{q} K^{-5 / 3}
\end{aligned}
$$

where $K$ is the wavenumber $\left(K=2 \pi n / V_{p}\right.$, with $n$ as the frequency in the aircraft time reference and $V_{p}$ the airspeed relative to the aircraft), $S$ is the spectral density, $\alpha_{i}$ is the Kolmogorov constant for the velocity ( $\alpha_{u}$ $=0.53$ for the longitudinal component and $\alpha_{v}=\alpha_{w}$
$=(4 / 3) 0.53$ for the other components $), \beta_{\theta}$ and $\beta_{q}$ are the constants for temperature and water content $\left(\beta_{\theta}\right.$ $=\beta_{q}=0.8$ ).

The inertial dissipation method was successfully tested over the sea: Large and Pond (1981, 1982), Fairall et al. (1988) present good comparisons between turbulent fluxes, calculated by this method and by the eddy-correlation one. For these comparisons, the data were measured on stable water towers. However, latent heat flux comparisons are scarce (Large and Pond 1982; Fairall et al. 1988). The inertial dissipation method is often used to calculate the drag coefficient and the transfer coefficients for sensible and latent heat (for example, see Fairall and Larsen 1986; Large and Pond 1981, 1982). Fairall and Markson (1987) used the inertial dissipation technique from aircraft low-level measurements over the sea; a comparison between friction velocity determined by this technique and by bulk method from a ship gave a good agreement.

\section{Application of airborne measurements}

This method was applied to low-level airborne measurements (generally $z<50 \mathrm{~m}$ ) during two experiments: one over the sea (TOSCANE-T experiment: Said 1988), the other in the Sahelian region with dry convective conditions (ECLATS experiment: Druilhet and Durand 1984). The measurements were made by the French research plane Hurel-Dubois 34 (Druilhet and Durand 1984). This aircraft enabled calculation of turbulent flux by the eddy-correlation method. These calculations have therefore been considered as the reference and have been compared with the calculations made using the inertial dissipation method explained above. Without entering into details of the aircraft's measuring equipment (Durand et al. 1985), we can point out that turbulent motion of the air with reference to the aircraft was measured by two mobile windvanes and a Rosemount 858 probe (attack and sideslip) and by a Pitot tube (airspeed of the aircraft), with these three sensors placed at the tip of a boom in front of the aircraft nose. Aircraft speed and angles of attitude were measured by an inertial navigation system and a doppler navigation radar. Turbulent fluctuation of air temperature was measured by a platinum-wire probe, type Rosemount 102E, with measurement correction according to the transfer function (Spyers-Duran and Baumgardner 1983; Guillemet 1983). This correction is of considerable size in the frequency band where the variance dissipation rate is calculated. Water mixing ratio fluctuations were measured by a Lyman alpha hygrometer, calibrated in absolute value against a dewpoint hygrometer.

Calculation of turbulent fluxes by the eddy-correlation method includes two kinds of errors: the measurement errors and the error due to the "method." For aircraft measurements, scalar fluctuations (such as temperature or moisture) are measured more easily 
than wind velocities, so that the measurement errors can be minimized. As far as the turbulent velocities of the air are concerned, their computation includes a lot of sensors: true airspeed (Pitot probe), attack and sideslip angles (in order to determine the air/aircraft velocities), and INS plus Doppler radar measurements (attitude angles, angular velocities, ground velocities), in order to calculate the aircraft/ground velocities. So, it is evident that the momentum flux calculation $\overline{u^{\prime} w^{\prime}}$ is less accurate than that of the sensible or latent heat. These errors are very difficult to evaluate, and the accuracy depends on the statistical behavior of the errors on each parameter.

The second kind of error results from the fact that the "true" flux should be calculated through a surface. In fact, it is calculated (from aircraft measurements) through a finite line. The accuracy $(\sigma)$ on a flux $(f)$ depends on (i) the measurement length $(l)$; (ii) the integral length scale of the process $(\mathcal{L})$; and (iii) the correlation between the turbulent functions $(r)$. This problem is discussed in Wyngaard $(1983,1986)$ and in Lenschow and Stankov (1986). They showed that a statistic estimation of this error can be expressed by

$$
\frac{\sigma}{f}=\left[\frac{2 \mathcal{L}}{l} \frac{\left(1+r^{2}\right)}{r^{2}}\right]^{1 / 2}
$$

For example, in our experiment over the sea (TOSCANE-T experiment, see following paragraph), $l$ is typically $20 \mathrm{~km}, \mathcal{L}$ averages $120 \mathrm{~m}$, and $r=0.30$ for momentum flux, 0.42 for sensible heat flux and 0.42 for latent heat flux (Said 1988). So, for momentum flux, $\sigma / f \sim 0.35$, whereas for sensible and latent heat flux, $\sigma / f \sim 0.26$. These values have to be taken into account for the following results.

For the TOSCANE-T experiment, ten flights were conducted. From these flights, about 60 low-level flight sequences were used, corresponding to moderately unstable conditions (the following ranges are determined from flight-averaged values): surface sensible heat flux varies between 5 and $50 \mathrm{~W} \mathrm{~m}^{-2}$, latent heat flux between 10 and $110 \mathrm{~W} \mathrm{~m}^{-2}$, friction velocity between 0.1 and $0.4 \mathrm{~m} \mathrm{~s}^{-1}$ and the Monin-Obukhov length between -400 and $-40 \mathrm{~m}$, which, given the flight altitude, corresponds to a stability coefficient of $(-z / L)$ from 0.1 to 1 . This is within the range of validity for the universal functions used. For the ECLATS experiment, 20 low-level flight sequences were analyzed. Sensible heat flux varied between 80 and $160 \mathrm{~W} \mathrm{~m}^{-2}$, latent heat flux between 20 and $90 \mathrm{~W} \mathrm{~m}^{-2}$, friction velocity between 0.2 and $0.6 \mathrm{~m} \mathrm{~s}^{-1}$, and the MoninObukhov length between -20 and $-140 \mathrm{~m}$, thus $-z$ / $L$ varies between 0.2 to 1 .

Computation of variance destruction rates was made in a frequency band ranging from 1 to $2 \mathrm{~Hz}$, which, given the aircraft speed, corresponds to range of wavelength from 30 to $60 \mathrm{~m}$. In view of the velocity, temperature, and moisture spectra analyzed during the campaign, it was apparent that this band was inside the inertial subrange. TKE dissipation rate is computed from the longitudinal (along the mean wind) or vertical velocity of the air. It must be noted that this computation uses data obtained from a lot of sensors, so that it is a priori less accurate than $N_{\theta}$ or $N_{q}$.

\section{Results}

Figures 1 to 3 present a comparison between turbulent fluxes calculated by the eddy-correlation method and the inertial dissipation method, for momentum flux, sensible heat flux and latent heat flux. The dissipation rate of TKE was calculated from the longitudinal wind component. For the three fluxes shown, there is good agreement between the two methods of estimation.

Sensible and latent heat flux comparisons are compatible with the accuracy given for eddy-correlation method. The linear correlation $(r)$ between the two methods has a value of $0.94(0.90)$, with a linear regression of $\overline{w^{\prime} \theta_{\text {diss }}^{\prime}}=0.79 \overline{w^{\prime} \theta_{\text {corr }}^{\prime}}+13\left(\overline{w^{\prime} q_{\text {diss }}^{\prime}}\right.$ $=0.85 \overline{w^{\prime} q_{\text {corr }}^{\prime}}+9$ ) for sensible (latent) heat flux. The rms of the absolute difference between the two fluxes estimations is $17.8 \mathrm{~W} \mathrm{~m}^{-2}\left(16.5 \mathrm{~W} \mathrm{~m}^{-2}\right)$ and the mean relative error is $45 \%$ (19\%) for sensible (latent) heat flux. High value for sensible heat flux must be attributed to the overestimation of low fluxes by the inertial dissipation method. These results are comparable to similar studies, such as those of Large and Pond (1982) of Fairall et al. (1988). However, it must be noticed that, at our knowledge, it is the first time that this comparison is made with aircraft data. A slight bias appears in sensible heat flux comparison. For low fluxes, the inertial dissipation method provides values greater than the eddy correlation one, whereas, for high fluxes, it provides lower values. However, the differences between the two methods remain within their accuracy

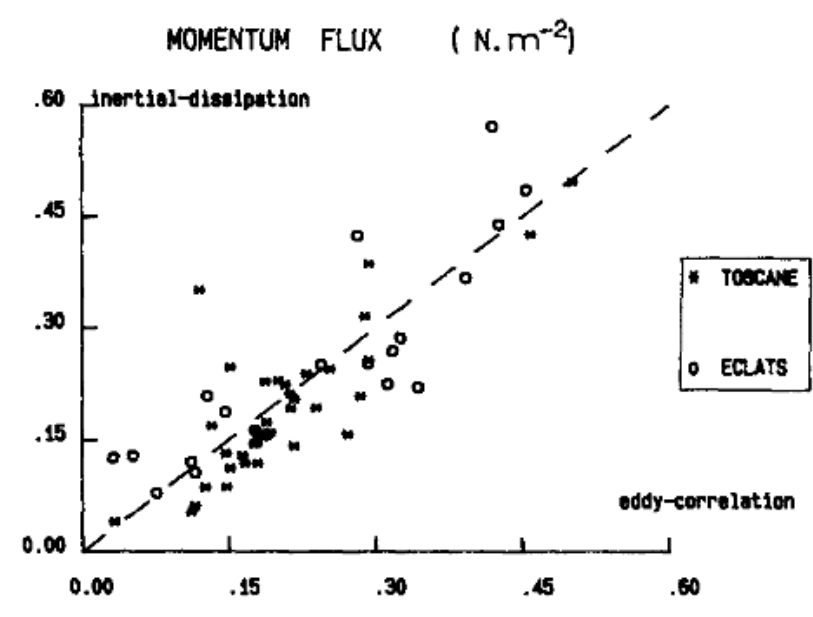

FIG. 1. Eddy correlation estimates of momentum flux $\left(\overline{u^{\prime} w^{\prime}}\right)$ versus inertial-dissipation estimates. 
SENSIBLE HEAT FLUX $\left(\mathrm{W} \cdot \mathrm{m}^{-2}\right)$

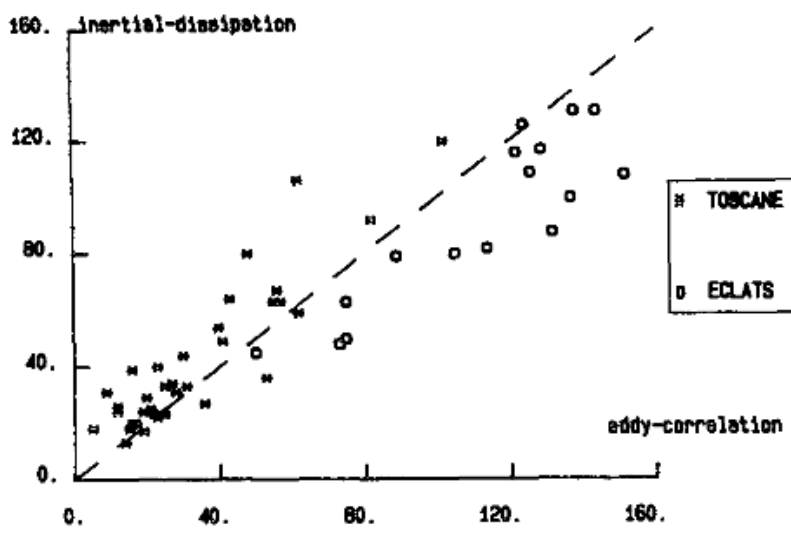

Fig. 2. Same as Fig. 1, but for sensible heat flux.

(except for very low values). It should be noted that an error in the calculation of $N_{\theta}$ could be due to the bad response of the temperature sensor in the frequency range used. This probe (Rosemount 102E2AL) presents an important attenuation at high frequencies (where computations are made). Such a problem is not present on the Lyman alpha hygrometer, and no bias appears on latent heat flux comparison. Turbulent transfer occurring at lower frequencies ( corresponding to some hundred meters in wavelengths), eddy correlation sensible heat flux should not be incriminated. But we must conclude that inertial dissipation method must be used with a fast response probe for temperature measurement.

Momentum flux comparisons provide weaker degree of agreement than those reported elsewhere (Large and Pond 1981; Fairall et al. 1988). The linear correlation between the two methods reaches a value of 0.84 , with a linear regression of: $\overline{u^{\prime} w_{\text {diss }}^{\prime}}=0.93 \overline{u^{\prime} w_{\text {corr }}^{\prime}}+0.013$. Given the data scattering, there is no significant bias. The rms of the absolute difference between the two fluxes estimations is $0.064 \mathrm{~N} \mathrm{~m}^{-2}$, and the mean relative error is $26 \%$. Considering the accuracy of the eddy-correlation method for momentum flux calculation (see above), this is not very surprising. Favorable comparisons for sensible and latent heat fluxes (for which the eddy-correlation method is more accurate than for the momentum one), could prove that the inertial dissipation method is a good method in order to estimate momentum flux from low-level airborne measurements.

\section{Towards a simplified method for calculating tur- bulent fluxes}

If calculation of $N_{\theta}$ and $N_{q}$ from airborne measurements does not seem to pose any particular problem, calculation of $\epsilon$, however, theoretically requires prior estimation of a turbulent wind component $u^{\prime}, v^{\prime}$ or $w^{\prime}$.
These calculations are complex, especially since precise knowledge of the frame of reference and the aircraft speed is necessary. The simplified formulations enabling this calculation are as follows (Lenschow 1986):

$$
\begin{gathered}
u^{\prime}=-V_{p} \cos (\Psi+\beta-A)+U_{a} \\
v^{\prime}=V_{p} \sin (\Psi+\beta-A)+V_{a}, \\
v^{\prime} \text { oriented } 90 \text { deg to the left of the mean wind } \\
w^{\prime}=V_{p} \sin (\alpha-\Theta)+W_{a}
\end{gathered}
$$

where $A$ is the angle of the direction of mean wind, $\Psi$ is the aircraft heading, $\alpha$ and $\beta$ are the attack and sideslip angles, $U_{a}, V_{a}$ and $W_{a}$ are the aircraft speeds in the mean wind frame, and $\Theta$ is the pitch angle. In these formulae, we can separate the terms that derive from motion of the plane itself, from those deriving from the movement of the air in relation to the plane. If the transfer function of the aircraft response to wind fluctuation becomes negligible before the inertial subrange frequency band where dissipation rates are calculated (as can be verified on aircraft movement spectra), the TKE dissipation rate can then be calculated in a simplified manner, only including the relative movement of the air in relation to the aircraft:

$u_{i}^{\prime} \approx\left\{\begin{array}{l}V_{p,}^{\prime} \text { if } \Psi-A \approx 0 \text { or } \approx \pi \\ \overline{V_{p}} \beta^{\prime}, \text { if }|\Psi-A| \approx \pi / 2 \text { or } \approx 3 \pi / 2\end{array}\right.$

$v_{i}^{\prime} \approx\left\{\begin{array}{l}\widetilde{V_{p}} \beta^{\prime}, \text { if } \Psi-A \approx 0 \text { or } \approx \pi \\ V_{p}^{\prime}, \text { if }|\Psi-A| \approx \pi / 2 \text { or } \approx 3 \pi / 2\end{array}\right.$

$$
w_{i}^{t} \approx \bar{V}_{p} \alpha^{\prime}
$$

where subscript $i$ denotes fluctuation in the inertial subrange. Figure 4 presents examples of power spectra of vertical velocity of the air, of the attack angle and

LATENT HEAT FLUX $\left(w . m^{-2}\right)$

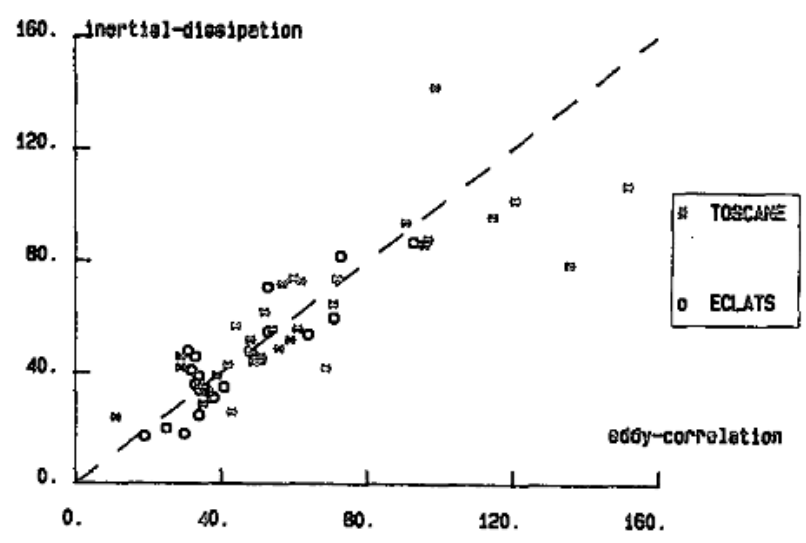

Fig. 3. Same as Fig. 1, but for latent heat flux. 

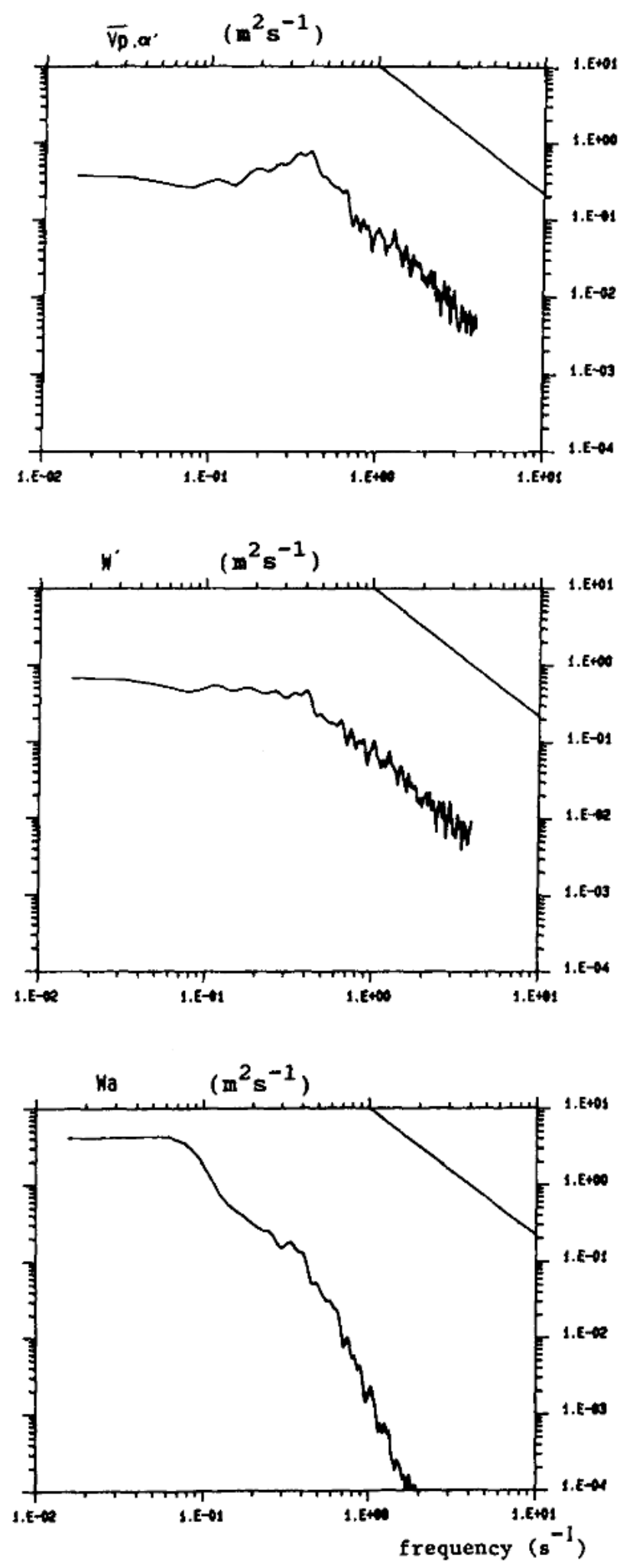

FIG. 4. From top to bottom: example of power spectra of the vertical velocity of the air $\left(w^{\prime}\right)$, of the angle of attack $\left(\alpha^{\prime}\right)$, and of the aircraft vertical velocity (Wa). Attack angle is multiplied by the average true airspeed, so that the three spectra are in the same units. Wa power spectrum values lower than $0.0001 \mathrm{~m}^{2} \mathrm{~s}^{-1}$ are removed. of the absolute vertical velocity of the aircraft $\left(W_{a}\right)$, as calculated from INS measurements. The sample is a turbulent horizontal leg $20 \mathrm{~km}$ long. It can be seen on the $W_{a}$ spectrum that the energy quickly decreases from $0.5 \mathrm{~Hz}$. In the inertial subrange, characterized by a $-5 / 3$ slope, the two spectra of $w^{\prime}$ and $\alpha^{\prime}$ show great similarity. Figure 5 shows the co-, gain, phase (cosine of) and coherence spectra between $w^{\prime}$ and $\bar{V}_{p} \alpha^{\prime}$. The gain between $x$ and $y$ is defined as

$$
G_{x y}(n)=\left[\frac{S_{x x}(n)}{S_{y y}(n)}\right]^{1 / 2}
$$

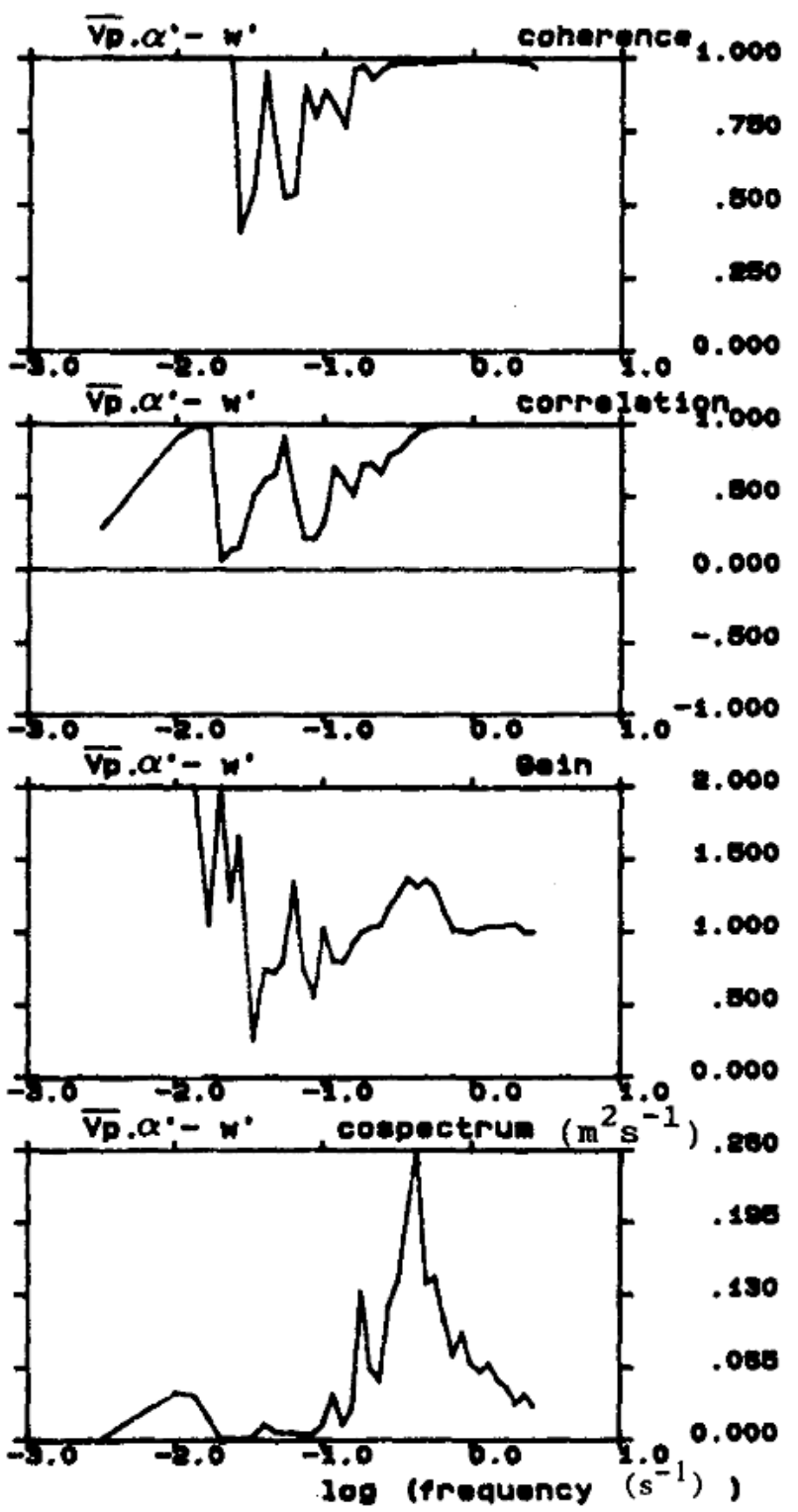

Fig. 5. From top to bottom: coherence spectrum, phase (cosine of) spectrum, gain spectrum and cospectrum between attack angle and vertical velocity of the air. The attack angle is multiplied by the average true airspeed, so that the two parameters are in the same unit. Same sample as for Fig. 4. 
where $S_{x x}$ and $S_{y y}$ are power spectra of $x$ and $y$. The coherence is very high above $0.2 \mathrm{~Hz}$, there is no phase shift between the two parameters above $0.5 \mathrm{~Hz}$, and the gain is very close to 1 above $0.7 \mathrm{~Hz}$. This figure demonstrates on an example that $\epsilon$ can be calculated solely from measurement of fluctuations in angle of attack and average airspeed. Figure 6 shows a comparison of $\epsilon$ as calculated from the vertical velocity of the air and calculated from the angle of attack. The frequency band chosen for the computation of $\epsilon$ is 0.8 $2 \mathrm{~Hz}$. Agreement is good and shows that this type of simplified method can be employed.

On the same manner, $\epsilon$ could be deduced from fluctuations in $V_{p}$, in which case however, it would also be necessary to know the relative angle of the wind with reference to the aircraft's heading, in order to adopt the value of Kolmogorov constant [see (10)]. If the wind is approximately parallel to the aircraft track, then $V_{p}$ is similar to $u^{\prime}$ and $\alpha_{u}$ must be used, whereas if the wind is approximately transversal, $V_{p}$ is similar to $v^{\prime}$ and $\alpha_{v}$ must be used. We do not present here these results, because the agreement is not as good as for $\alpha^{\prime}$ (Fig. 6). In particular, the comparison between $\epsilon$ calculated from longitudinal wind component, and from $V_{p}$ fluctuations presents differences of about $15 \%$. This would introduce an error of about $10 \%$ on the momentum flux [assuming conditions close to neutrality in Eq. (7)]. One of the reasons is that the Kolmogorov' constant chosen, $\alpha_{u}\left(\alpha_{v}\right)$, is theoretically valid only along (perpendicular to) the mean wind. In reality, the sampling direction is anywhere. Note that this problem does not appear when the attack angle is used.

\section{Conclusion}

We have seen that the inertial dissipation method, when used for airborne measurements at low altitudes,

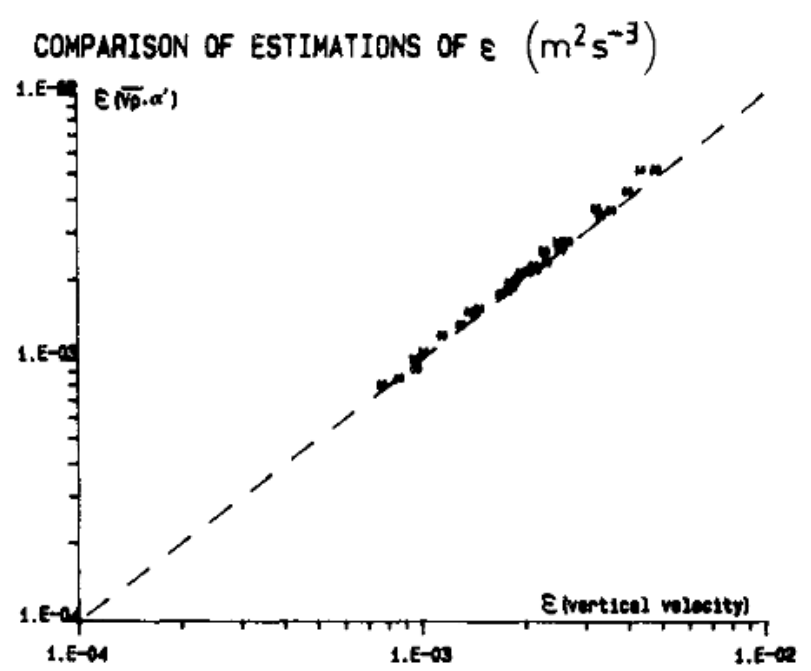

FIG. 6. TKE dissipation rate, deduced from the vertical velocity of the air versus that deduced from the angle of attack. allows good accuracy in the calculation of turbulent flux in the surface layer, for moderately unstable conditions. In particular, this work confirms the ability of the inertial dissipation method to calculate the latent heat flux in a wide range of values. There are very few similar results in the literature. The agreement between this method and the eddy correlation one is better for sensible and latent heat fluxes than for the momentum flux. This could be due to the inaccuracy in eddy-correlation calculations (inaccuracy in wind components calculation with aircraft and low values of momentum flux correlation). Thus, favorable comparisons for sensible and latent heat fluxes prompt us to use the inertial dissipation method in order to calculate the momentum flux from low altitude aircraft measurements.

We proposed then a simplified method in order to calculate TKE dissipation rate from one sensor only with the same accuracy as the exact method. It can be calculated simply from measurements of fluctuations in the angle of attack, or from fluctuations in aircraft airspeed. In the latter case, however, the relative orientation of the aircraft with respect to the mean wind must be known, and the results are not as good as for the attack angle.

This method is interesting by its great simplicity of implementation as compared with the classical eddycorrelation method. However, it must be verified that the transfer function of the aircraft response becomes negligible before the inertial subrange frequency band chosen for the calculations. Therefore, the frequency range used for TKE dissipation rate calculation depends on the inertial aircraft characteristics (the smaller the aircraft, the higher the frequency range).

Lastly, this method is particularly well adapted to calculating flux at the ocean-atmosphere interface, where conditions of stability are generally moderate.

Acknowledgments. Special thanks are due to the "Division Technique" of the "Institut National des Sciences de l'Univers" who performed the measurements aboard the aircraft, and to the "Institut Geographique National" who operated the Hurel Dubois 34 aircraft. This work was financially supported by the INSU of the "Centre National de la Recherche Scientifique" of France and by the "Conselho Nacional de Desenvolvimento Cientifico e Tecnologico" (CNPq) of Brazil.

\section{REFERENCES}

Druilhet, A., and P. Durand, 1984: Study of the sahelian convective boundary layer with dust haze (ECLATS experiment). Bound.Layer Meteorol., 28, 51-77.

Durand, P., F. Said and A. Druilhet, 1985: Airborne measurements applied to the determination of boundary conditions over the sea-TOSCANE experiment. Proc. Third Int. Collog. on Spectral Signatures of Objects in Remote Sensing, Les Arcs, France, 2328.

Dyer, A. J., and B. B. Hicks, 1970: Flux-gradient relationships in the constant flux layer. Quart. J. Roy. Meteor. Soc., 96, 715-721. 
